\title{
The effects of delay and exposure duration in a face recognition task
}

\author{
GAIL J. WALKER-SMITH \\ University of Oxford, Oxford, England
}

\begin{abstract}
Face recognition was investigated in a successive comparison task. Subjects were required to make same/different judgments about pairs of Photo-fit faces that were either identical or differed by a single feature. Picture information extraction and retention were examined by manipulating stimulus delay and exposure duration. Results indicated that overall performances was better for the top of the face. The eyes and mouth were more vulnerable than the rest of the face to recognition decrement after a delay, possibly due to their role in facial expression. When features were ranked in order of processing difficulty for each subject, it appeared that features were processed serially and delay affected a retrieval stage, while short exposure affected a visual comparison stage of processing. For the feature ranks, a single dimension of "salience" appeared to be both perceptual and mnemonic.
\end{abstract}

Dramatic empirical evidence (e.g., Nickerson, 1965; Shepard, 1967; Standing, Conezio, \& Haber, 1970) has shown that people have a strikingly good recognition memory for pictures. However, this performance is highly variable across studies, due partly to the nature of the experimental material (see Goldstein \& Chance, 1974, for a review). Recognition scores can be depressed by using homogeneous sets of stimuli (e.g., Goldstein \& Chance, 1971; Scapinello \& Yarmey, 1970), but it is also important to consider the type of stimuli that are employed in experiments because, as Goldstein and Chance (1974) state, different classes of picture are unequal in "memoriability."

The present experiment was conducted in order to investigate recognition memory for a specific set of pictures-namely, faces-which have been regarded as a "psychologically special class of stimuli" (Ellis, 1975, p. 410). The aim was to explore visual information extraction and retention in a successive face recognition task by manipulating stimulus exposure duration and the retention interval.

Are faces perceived and stored holistically, or are they analyzed into separable features? A remarkable picture memory performance need not imply that detailed pictorial information is held in memory, since usually only partial recognition is necessary in order for correct responses to be made. Therefore, although people make identification judgments about a whole

This article is based upon research for a doctoral thesis, which was supported by the British Science Research Council. The author is most grateful to Anne Treisman, her supervisor, for her invaluable advice throughout the research and preparation of this manuscript, and to Gillian Cohen for her helpful comments on an earlier draft of the paper. Requests for reprints should be addressed to G. J. Walker-Smith, Department of Experimental Psychology, University of Oxford, South Parks Road, Oxford, OX1 3UD, England. face, their responses could be based on the comparison of salient features. In fact, people do find some areas of the face more important than others for identification (Ellis, 1975).

One method of determining the relative importance of the different areas of the face is to examine performance across facial features in a face comparison task where subjects are required to detect alterations in specific features. This was attempted in the present study. By constructing pictures of faces from a Photo-fit Kit, ${ }^{1}$ five components-the forehead and hair, the eyes and eyebrows, the nose, the mouth, and the chin-can be experimentally manipulated. These features provide a convenient way of dividing the face into subpatterns without necessarily implying that they possess psychological reality as perceptual features or that they are processed as independent units.

Two kinds of question regarding the processing of facial features are of interest. The first concerns which features are most important for identification. Conflicting results have emerged from the literature (see Davies, Ellis, \& Shepherd, 1977, for a brief review). However, Walker-Smith, Gale, and Findlay (1977) reported eye-movement data which indicates that different subjects concentrate on different facial features, so this may account for some of the discrepant findings. One aim of this experiment was, therefore, to explore further the question of whether some features are more salient. The second question involves cancelling out differences in particular feature preferences across individuals by rank ordering, so that one can ask: How does the ease or difficulty of processing a feature affect performance across different kinds of task? (For example, is feature processing serial for one task and parallel for another?)

It has been reported that face recognition is not affected by a delay (Laughery, Fessler, Lenorovitz, 
\& Yoblick, 1974; Yarmey, 1971), but in these experiments recognition was not tested immediately after presentation, when performance could have been enhanced by information stored in a short-term visual memory. Phillips and Baddeley' (1971) demonstrated that forgetting occurred over a 9-sec interval for difficultto-name visual patterns that were masked to minimize iconic storage effects. Also, Smith and Nielsen (1970) found that error rates increased over a $10-\mathrm{sec}$ retention interval for schematic faces. Therefore, it was predicted that more recognition errors would be made after a delay was imposed between a target and test face, in comparison with immediate testing. Furthermore, the following experiment sought to determine (1) whether changes in each part of a face that can be detected with immediate testing can also be recognized after a delayed test, when any short-term visual memory advantage is reduced, and (2) whether features that are found to be most salient initially are also better memorized.

Bradshaw and Wallace (1971) reported data from a simultaneous face comparison task, using Identi-Kit pictures, which suggested that subjects used a serial selfterminating feature comparison strategy. Similarly, Smith and Nielsen claimed that their schematic faces were processed by self-terminating feature comparisons when different judgments were being made in a sequential comparison task. So, if the most salient features are not only the features that are easiest to discriminate but are also the features that are processed first, response latencies should correlate with error rates. Thus, a difference in a salient feature dimension should result in a quick response latency. These measures could, however, conflict. Hence, in this study, both these dependent measures were examined to see whether they correlated or whether subjects adopted a speederror tradeoff.

Another method of investigating how pictures are processed and of judging the relative importance of their various components is to reduce the presentation time of one of the stimuli in a successive comparison task. In this study, the exposure duration of the test face was reduced in some experimental conditions, thus limiting the amount of information that could be extracted from the face. Given limited processing time, it was expected that only salient parts of the stimulus would be encoded, so that accurate recognition responses would only be made when these salient features were altered in the test face.

From the change in error rates when the interstimulus interval is lengthened, it should be possible to infer the order in which different features are lost, while the change in error rates when exposure is reduced should indicate the order of feature extraction. By comparing the effects of these two manipulations across features, one can discover whether there is a single dimension of "salience" which is both perceptual and mnemonic. For, if one exists, there should be a positive correlation across features between the rate of extraction and their retention.

\section{METHOD}

\section{Subjects}

Eight undergraduates, five male and three female, served as subjects and were paid for participating.

\section{Materials}

Three examples of forehead and hair (F), eyes and eyebrows (E), noses $(\mathrm{N})$, mouths $(\mathrm{M})$, and chins $(\mathrm{C})$ were selected from the five feature dimensions employed in the Photo-fit Kit. The hairstyles were chosen from a subset of hair that was short and dark, and the other features were randomly selected from the kit after features with unusual characteristics had been eliminated. A single front-view, male, Caucasian face was constructed from one set of features. The remaining two examples were used to construct 50 variations of the face -10 where one dimension was altered and 40 where all combinations of two features were changed. Thus, a pool of 51 faces was generated, and from this set it was possible to select pairs of faces where only one feature was altered (i.e., F, E, N, M, or C). These stimuli were presented as black-and-white photographs of heads, measuring approx imately $65 \times 45 \mathrm{~mm}$ and centered on white cards. When displayed in a tachistoscope, they subtended approximately $7^{\circ} \times 5^{\circ}$ of visual angle (equivalent to viewing a real head at a distance of about $2 \mathrm{~m})$.

An additional tachistoscope card was fabricated. This was a pattern mask which consisted of a mosaic made from parts of a face picture which had been cut into small pieces. Finally, a further set of 10 faces was made to use as practice stimuli.

\section{Procedure}

Subjects were run individually for four $1 \frac{11 / 2-h}{\text { sessions. Each }}$ session comprised a single condition, and the session order was counterbalanced across subjects.

Subjects viewed the stimuli through a three-field tachistoscope. Each session was started with a practice run of 20 trials, and then 90 experimental trials were administered. For each experimental session, nine pairs of stimuli were chosen where one of the five feature dimensions altered. The nine pairs for each dimension were selected at random from the stimulus pool, with the restriction that the three values assumed by each facial feature be combined in equal proportions (e.g., where the eyes altered between the two faces, there would be three cases where E1 was paired with E2, three cases where E1 was paired with E3, and three cases where E2 was paired with E3, etc.); the order of the paired stimuli was randomized. Forty-five presentations of pairs of stimuli that altered by one feature dimension (nine each of $F, E, N, M$, and $C$ ) were randomly mixed with 45 presentations of identical pairs. For each session, all but a random selection of six faces from the stimulus pool were presented twice to form identical pairs. Thus, in the recognition task there were equal numbers of same and different face pairs.

After the experimenter had warned the subject of the commencement of a trial, the first face of a pair (the target) was presented for $2 \mathrm{sec}$. The target was immediately followed by the pattern mask. In Condition 1 (control) and Condition 4 (short exposure), the mask was presented for $1 \mathrm{sec}$. In Conditions 2 (long delay) and 3 (long delay and short exposure), the mask remained on the screen for $20 \mathrm{sec}$ and a warning tone was sounded for $1 \mathrm{sec}, 1 \mathrm{sec}$ before the next stimulus (the test face) was displayed. The test face immediately followed the mask. It was exposed for $1 \mathrm{sec}$ for Conditions 1 and 2 but for only $65 \mathrm{msec}$ for Conditions 3 and 4 . This face was again immediately followed by the mask, which was displayed for $1 \mathrm{sec}$.

The subjects were instructed to respond "same" or "different" as quickly and as accurately as they could by pressing the appropriately labeled response switches, which stopped a digital timer started by the onset of the test stimulus. For half the subjects, the same key was on the right and the different key was on the left, while the other half had the opposite arrangement. In the conditions where a delay was imposed between the two stimuli, a three-figure number was read to the subjects from 
random number tables at the onset of the interstimulus interval. The subjects were then required to count backwards in threes to prevent verbal rehearsal of the target face until the onset of the warning tone.

The subjects were told that on about half the trials the second face would be the same as the first, while for the other trials the second face would alter from the first by a single change in one of the five features. After the subjects had made their same/different judgment, they were asked to rate their response on a 4-point scale to indicate how confident they were that their judgment had been correct $(1$-confident; 2 -fairly confident; 3 -not very confident; 4-guess).

\section{Design and Analyses}

Correct reaction times, confidence ratings for correct responses, and error rates were the dependent measures recorded in this study. Separate analyses of variance were performed for each type of data, and for these analyses only correct different responses and their associated error rates were considered.

In the facial features analyses of variance, the independent variables-feature altered $(F, E, N, M$, and $C$ ), exposure duration (long, short), and delay (long, short)-were factorially combined in an 8 (subjects) by 5 by 2 by 2 randomized blocks design.

In an attempt to reduce any Subjects by Features individual differences, the data were reanalyzed using a rank order procedure. For each subject and for each dependent measure, ranks from 1 to 5 were assigned to facial features in ascending order of task difficulty encountered in the control condition. For this purpose, it was assumed that decreasing confidence, increasing error rates, and slower response latencies reflect increasing task difficulty. Where control condition scores were tied, they were rank ordered to fall in line with the overall mean control condition results. A second set of analyses of varianice was then carried out using feature ranks as an alternative parameter to facial features. Here, the observations from the experimental conditions were aligned with each subjects' control condition rank so that it could be determined whether the experimental manipulations differentially affected "easy" or "difficult" components of the face.

In addition, a set of rank order analyses were performed where confidence ratings and error rates were aligned with each subjects' reaction time rank in the control condition, to show how the three measures covaried.

\section{RESULTS}

\section{Facial Features Analyses}

The results of these analyses are presented graphically in Figure 1.
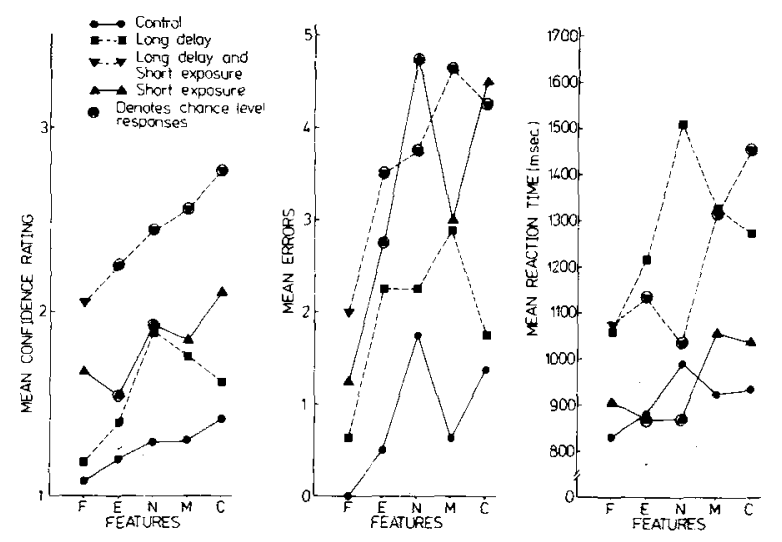

Figure 1. Mean confidence ratings, error rates, and reaction times for the facial features analyses.
All three dependent measures revealed significant effects for the feature altered: confidence ratings, $F(4,28)=5.48, p<.01$; reaction time, $F(4,28)=3.54$, $p<.05$; errors, $F(4,28)=4.39, p<.01$. It seems that the five features are not equally important for recognition. The hair region and, to a lesser extent, the eye region appear to be more quickly, accurately, and confidently processed than the other facial features. Thus, performance for the top of the face may be superior to that for the bottom. This possibility was supported by significant results from Scheffé tests, where, for $F$ and $\mathrm{E}$ vs. $\mathrm{N}, \mathrm{M}$, and $\mathrm{C}, \mathrm{p}<.01$ for confidence ratings, $\mathrm{p}<.05$ for reaction time, and $\mathrm{p}<.05$ for errors.

There was a significant main effect of exposure duration for confidence ratings, $F(1,7)=19.90, p<.01$, and errors, $F(1,7)=40.60, p<.01$, but not for reaction time. Confidence was lower and the error rate was higher for the $65-\mathrm{msec}$ exposure than for the $1-\mathrm{sec}$ exposure. Yet no consistent increase in reaction time was found across subjects from the long to the short exposure duration. However, the Feature by Exposure Duration interaction was found to be significant for the reaction time data, $F(4,28)=3.47, \mathrm{p}<.05$, but not for confidence ratings or errors. Pairwise comparisons using Tukey's HSD tests (Kirk, 1968) (all subsequent pairwise comparisons are made using this test) show that the average latencies for the $F, E, M$, and $C$ did not significantly differ from the long to the short exposure, but for $\mathrm{N}$ there was a significant decrease in latency after the short exposure, $p<.01$. Subjects were less likely to be correct after a short exposure regardless of the changed feature, but if subjects made a correct response, the mean reaction time was constant across exposures. The data suggest that exposure duration affects a nonspecific stage of processing for accuracy and confidence and affects a feature-specific stage for comparison time.

The main effect of delay gave significant results for confidence ratings, $F(1,7)=12.25, \mathrm{p}<.01$; reaction time, $F(1,7)=13.72, p<.01$; and errors, $F(1,7)=$ $17.54, \mathrm{p}<.01$. Confidence decreased, more errors occurred, and average response latencies were longer following the 20 -sec delay. The only significant Features by Delay interaction was for the error rates, $F(4,28)=$ $4.21, \mathrm{p}<.01$. Relatively few errors occurred after both short and long delays for the $F$ alteration, while error rates remained high for the $N$ and $C$. The $E$ and $M$ received high error rates after the 20 -sec delay, but performance was significantly better at the short delay, $\mathrm{p}<.01$.

No additional significant effects were found.

\section{Feature Rank Analyses}

Figure 2 shows the graphed feature rank data.

The main effect of rank was significant for confidence ratings, $F(4,28)=7.62, \mathrm{p}<.01$; reaction time, $\mathrm{F}(4,28)=2.88, \mathrm{p}<.05$; and errors, $\mathrm{F}(4,28)=8.85$, $\mathrm{p}<.01$. Performance declined as the rank increased (with one nonsignificant exception-more errors were made for the third than for the fourth rank). 
The effect of exposure duration was again significant for confidence ratings, $F(1,7)=19.90, p<.01$, and errors, $F(1,7)=40.60, p<.01$, but not for reaction time. Confidence decreased and errors increased from the long to the short exposure durations. Also, the main effect of delay was significant for all three dependent measures: confidence ratings, $F(1,7)=12.25$, $\mathrm{p}<.01$; reaction time, $\mathrm{F}(1,7)=13.72, \mathrm{p}<.01$; and errors, $F(1,7)=17.54, p<.01$. Confidence decreased and latencies and errors increased from the short to the long delay. Unlike the feature analyses, no significant interactions were discovered-feature rank did not interact with exposure duration or delay.

In order to consider the dependent measures in comparison with each other, a further set of analyses was carried out where all the data was rank ordered for each subject in the same order as the latency data for the control condition. The analyses of variance here gave similar patterns of significant results to the aforementioned ranks analyses of variance.

The latencies for the ranked data form linear functions for both the control and the delay conditions. Linear regressions were calculated for these conditions (control: $y=704.28+68.87 x$; delay: $y=1101.37+$
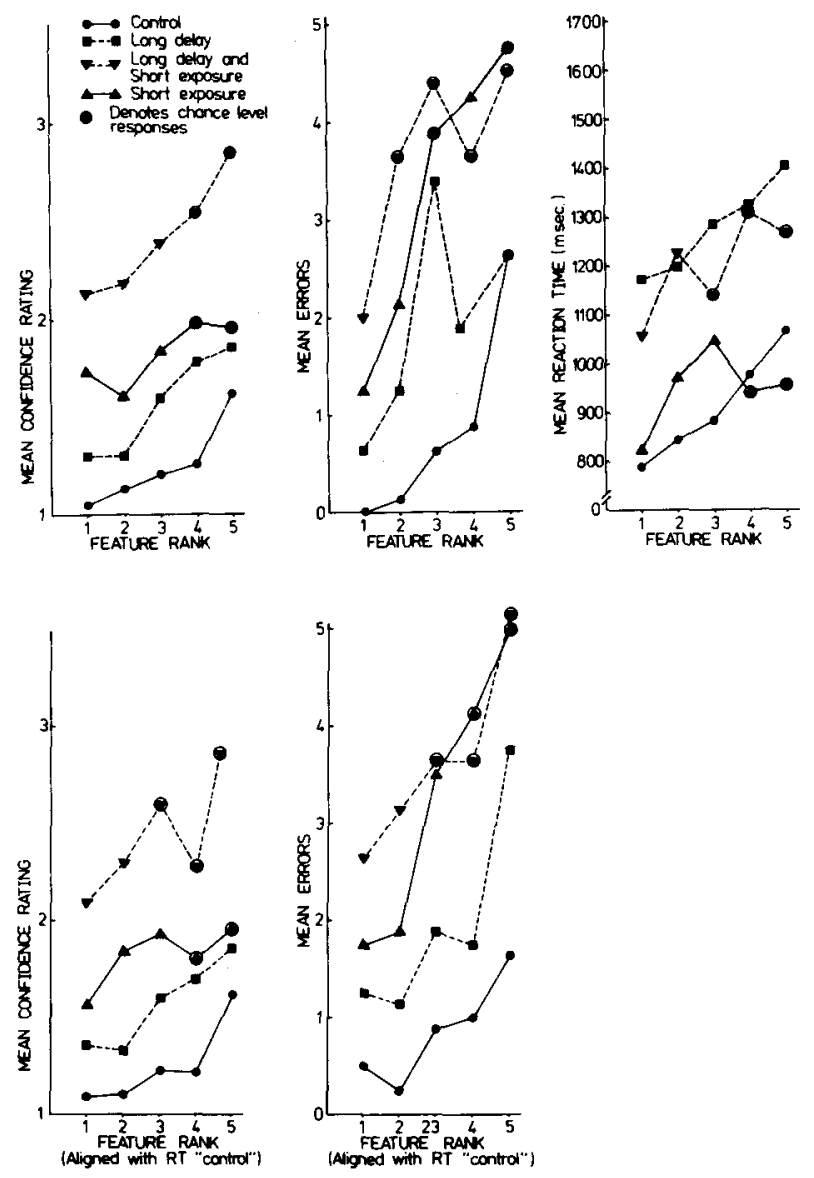

Figure 2. Mean confidence ratings, error rates, and reaction times for the feature ranks analyses.

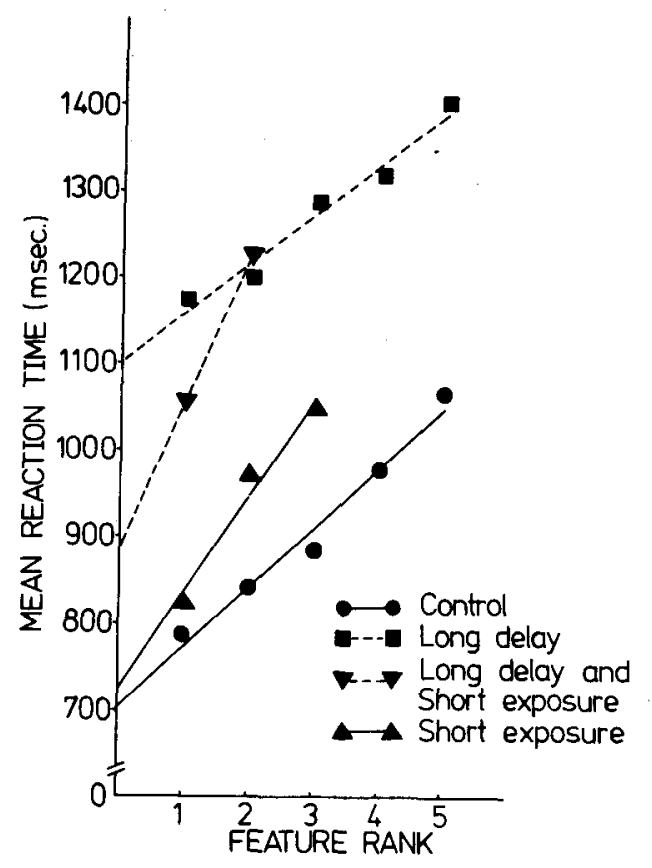

Figure 3. Mean reaction times and regression curves for the feature ranks analysis. (Number of data points per mean is at least 41, maximum is 72; mean scores for responses no better than chance level accuracy are excluded.)

$58.33 \mathrm{x}$ ), and they each account for $97 \%$ of the variance due to rank order (see Figure 3). The gradients fell within the standard errors of each other, so it is concluded that the two lines could be considered parallel with different intercepts. The same rank order of processing appears to be operative after the $20-\mathrm{sec}$ delay, but - in addition to the time required to process each feature (approximately $65 \mathrm{msec}$ per feature), after the delay, a time constant of between 355 and 439 msec needed to be added to the processing time for each feature rank. When the mean different latencies for the control and delay conditions were projected onto these regression lines, they suggested that just over 2.5 features were processed on the average before a different response was made-if serial processing is assumed.

Figure 3 also includes linear regression curves for Conditions $3(y=888.63+168.75 x)$ and $4(y=722.88$ $+112.31 \mathrm{x})$. These were calculated from two and three data points, respectively, since performance appears to be no better than chance for the higher feature ranks (see below). The intercept for the short-exposure condition was similar to that for the control condition (control: $704.28 \pm 22.32$; short exposure: $722.88 \pm$ 45.14 ), yet the gradient was steeper, while for the short exposure and delay condition, both the intercept and gradient differed from those of the control condition. Less reliance is placed upon these two results than on those for Conditions 1 and 2, first, because the curves were calculated from so few data points and, second, because the data points were based on fewer raw scores as error rates were high. 


\section{General Feature and Rank Results}

For observations ordered by feature and feature rank, the reaction time data appeared to point to anomalous results. For example, for the most part, response latencies in Condition 3 were shorter than those for Condition 2, and for some features and feature ranks reaction times after the short exposure condition were even shorter than the control latencies. It was suspected that these short latencies were the result of correct guessing. In order to detect data points where the number of correct responses was no greater than chance, a series of $t$ tests was carried out on the feature and feature rank error rates (where the ranking was in accordance with the reaction time control condition) after performing arcsin transformations. Each of the five mean data points per condition was tested against expected mean values, which were derived by means of a chi-square procedure. Since same responses could not be subdivided into feature or rank data, one-fifth of the total errors per condition for same responses was used as a parameter and, together with the different response data for each point, a contingency table was constructed. These tests indicated that, for the features analysis and the ranks analysis, all correct responses in the control and delay conditions were greater than that expected by chance. In the features analysis, in the short exposure condition correct responses for $\mathrm{E}$ and $\mathrm{N}$ were at chance level, while for the delay plus short exposure only correct responses for the $F$ were significantly better than chance. For the ranked data, in the shortexposure condition responses for the fourth and fifth feature ranks were no better than chance, and in the delay plus short-exposure condition only the first and second features had significantly fewer errors than chance.

Finally, Pearson product-moment correlation coefficients were calculated to indicate how the dependent measures covaried. Table 1 shows the results. For the overall feature and feature rank (ordered by reaction time control) data, the measures correlated significantly-as errors increased, both speed and confidence decreased. When only the control and delay conditions were considered, the correlation coefficients were greater for the ranked data than for the feature data, $t(5)=3.05, p<.05$.

\section{DISCUSSION}

\section{Facial Features}

Significant differences in confidence, accuracy, and speed of detecting alterations of particular facial features imply that some parts of the face are more salient than others. The fact that the forehead and hair alteration was significantly more accurately detected than the other features (and more confidently and more quickly detected than some of the others), corroborates Goldstein and Chance's (1971) subjective data from people whose modal report was that they believed that the, hair was the main feature that helped them to remember faces. For each dependent measure, the hair received the best performance, followed by the eyes. Thus, performance for the top of the face was superior to that for the bottom. Goldstein and Mackenberg's (1966) results with children were similar. They found the upper half of faces more useful than the lower half as recognition cues. The present results also support Davies et al.'s (1977) findings. They showed that the hair and then the eyes were the most accurately recognized features of their Photo-fit stimuli.

It was predicted that if face recognition were to be tested after different retention intervals, performance would be worse following longer interstimulus intervals. In fact, all analyses showed that delay was effective in changing subjects' performance. Overall picture forgetting did occur over the filled interval. The backward counting task prevented verbal rehearsal during the 20-sec delay, but no specific verbal rehearsal prevention task was employed during the short interstimulus interval. Therefore, it is possible that the performance decrement after the long delay can be accounted for merely by the loss of the verbal component of memory, thereby increasing the error rate and decreasing confidence. Latencies could be slower because of the processing switch from counting to picture comparison together with the search time needed for locating the stored face. Alternatively, it is possible that the initial 2 -sec presentation is not long enough to allow an adequate verbal description to be made which can distinguish one face from another. The pattern mask would prevent the use of iconic information at the short delays. Thus, the two levels of performance may reflect the loss

Table 1

Pearson Product-Moment Coefficients for Correlations Between Reaction Time (RT), Confidence Rating (CR), and Error Rate (ER)

\begin{tabular}{|c|c|c|c|c|c|c|c|c|c|c|c|c|c|c|}
\hline & \multicolumn{4}{|c|}{ Overall } & \multicolumn{4}{|c|}{ Control Condition } & \multicolumn{4}{|c|}{ Delay Condition } & \multirow{2}{*}{\multicolumn{2}{|c|}{$\begin{array}{c}\cdot \\
\text { Overall Mean } \\
\text { Rank Data } \dagger \dagger\end{array}$}} \\
\hline & \multicolumn{2}{|c|}{$\begin{array}{c}\text { Mean Features } \\
\text { Data } \\
\end{array}$} & \multicolumn{2}{|c|}{$\begin{array}{c}\text { Mean Rank } \\
\text { Data } \dagger\end{array}$} & \multicolumn{2}{|c|}{$\begin{array}{c}\text { Mean Features } \\
\text { Data }\end{array}$} & \multicolumn{2}{|c|}{$\begin{array}{c}\text { Mean Rank } \\
\text { Data } \dagger\end{array}$} & \multicolumn{2}{|c|}{$\begin{array}{c}\text { Mean Features } \\
\text { Data }\end{array}$} & \multicolumn{2}{|c|}{$\begin{array}{c}\text { Mean Rank } \\
\text { Data } \dagger\end{array}$} & & \\
\hline & CR & ER & $\mathrm{CR}$ & ER & CR & ER & CR & ER & CR & ER & CR & ER & CR & ER \\
\hline $\begin{array}{l}\text { RT } \\
\text { CR }\end{array}$ & $.96 * *$ & $\begin{array}{l}.87^{* *} \\
.91^{* *}\end{array}$ & $.87 * *$ & $\begin{array}{l}.87 * * \\
.97 * *\end{array}$ & $.76^{*}$ & $\begin{array}{l}.94^{* *} \\
.78^{*}\end{array}$ & $.90 * *$ & $\begin{array}{l}.92^{* *} \\
.94^{* *}\end{array}$ & $.96 * *$ & $\begin{array}{l}.70^{* * *} \\
.76 *\end{array}$ & $.99 * *$ & $\begin{array}{l}.91 * * \\
.88 * *\end{array}$ & $.90 * *$ & $\begin{array}{l}.93 * * \\
.90^{* *}\end{array}$ \\
\hline
\end{tabular}

$* p<.05 \quad * * p<.01 \quad * * *$ Nonsignificant.

tordered by $R T$ control. $\quad$ f tordered by independent control condition data. 
of visual information over time. [This possibility is supported by results from a later experiment (in preparation), where a similar performance deficit was also found when subjects had no interference task during a 20 -sec delay.] The fact that rank orderings were so similar and the latency functions were parallel at the two delays suggests a unitary representation rather than two separate memory stores.

Error rates were less than chance for all feature dimensions for both the control and the long-delay conditions. Thus, it is possible to recognize each part of the face after a long delay, but the accuracy of performance after each interstimulus interval depends upon the feature altered. Although no significant differences in errors occurred for the $\mathrm{F}, \mathrm{N}$, or $\mathrm{C}$, at the two intervals, both the $\mathrm{E}$ and $\mathrm{M}$ received fewer errors at the short delay, yet after $20 \mathrm{sec}$, accuracy was similar to that for the $\mathrm{N}$ and $\mathrm{C}$. Thus, there was a significant short-term recognition advantage for the eyes and mouth. Accuracy for the $F$ was highest after both delays, although $F$ (long) was not significantly more accurate than $\mathrm{E}$ (short) or $\mathbf{M}$ (short). Ignoring any individual differences, the evidence suggests that the hair is the best registered feature of the face and its importance is not lessened after a delay. This is perhaps ecologically useful, because in natural conditions the hair may provide the best recognition cue at distances where the internal facial features are indistinct, thus allowing a person to be recognized further away from an observer than would be the case if the whole face had to be clearly visible first. But why should the mouth and eyes be recognized accurately at short delays but not after long intervals? Michael Argyle (1975, p. 211) tells us that the face is the most important area for nonverbal communication, and within the face the eyes and mouth regions are the most mobile areas-they change in appearance and continually alter the signals they send. Therefore, over a short period, it would be useful to store specific feature arrangements so that observers could monitor changes in expression and thus follow nonverbal signaling. However, in long-term memory, it is improbable that the whole gamut of a particular person's facial expressions are stored. Instead, only a generalized representation of the face would be necessary for person recognition. So, the Features by Delay interaction results could be explained by suggesting that at the short delay detailed information is stored so that expression comparisons can be made, but after a long delay less information is available so that person recognition may take place but expressional nuances are forgotten.

Performance decreased from the long to the short exposure duration, with the exception of latency measures for detecting changes in the eyes and nose. When the long delay was combined with the short exposure, only the forehead and hair received fewer errors than chance, and in the short-exposure condition more errors than chance were made when detecting alterations in the eyes and nose. Therefore, the significant Features by Exposure Duration interaction for the latency data possibly results from the inclusion of a large proportion of correct guesses in the data. The only correct responses greater than chance for both short exposure conditions were made for the hair, which suggests again that this feature was processed earlier than the rest of the face. The short stimulus duration resulted in a ceiling effect so the only fixed feature ordering that can be determined at the picture information extraction stage is that processing for the hair terminates first.

\section{Feature Ranks}

The feature ranking reaction time analysis gives parallel functions for the control and long-delay conditions. According to a Sternberg $(1967,1969)$ additive factor method for using latency measurement to study stages of information processing, this suggests that a serial processing strategy was used in the control condition [although it need not necessarily follow, since Townsend (1971) argues that parallel systems could also predict this result]. This type of processing was also found after the long delay, with the addition of an extra process which required approximately $400 \mathrm{msec}$ for completion. The increase in the delay condition intercept in comparison with the control could be due to a fixed time that is needed to switch from the counting task to the face comparison. An alternative explanation is that it takes about $400 \mathrm{msec}$ to retrieve the initial face from longterm storage and to have the information in a suitable form so that serial feature comparisons between it and the second picture can be made.

In the short-exposure condition, it is difficult to make any strong inferences from three points, but it seems that serial processing is also indicated. The intercept was not significantly different from the control condition, although the gradient of the function differed. Thus the short exposure may affect the same processing stage as the delay. The hypothetical face retrieval stage took the same length of time as it did in the control condition, but instead of about $65 \mathrm{msec}$ being needed to scan each feature, approximately $110 \mathrm{msec}$ was required. The increased feature comparison time in Condition 4 may, perhaps, be due to the poorer quality of the encoded test face and the difficulty encountered in matching it with the target. Sternberg (1967) argued that an increased slope, following visual degradation (he used visual noise), is evidence for a visual, as opposed to verbal, comparison operation. However, since the error rates were high and variable here, one must be cautious when arguing from latencies which may not be very reliable.

The feature rank results suggest that when different responses are made, features are processed in a fixed order both immediately and when information has to be retrieved from a more permanent memory store. Since 
similar results were not exhibited in the feature analysis, it is possible that there are individual differences in the particular facial feature processing priorities that subjects adopt, which masks this finding.

Overall performance tended to decrease as the rank increased with one nonsignificant exception. However, when errors were rank ordered according to the latency control data, errors increased from Ranks 1 to 5 . The ranking procedure is possibly more reliable for the reaction time data, because in the error data there were many tied scores in the control condition. Therefore, each subjects' tied scores had to be ranked by making feature error rates fall in line with the overall mean control condition feature rank. There were significant positive correlations between the three dependent measures for the overall ranked data and for the feature results too. Therefore, there is evidence that some parts of the face are processed more quickly and more accurately than others. These features are also those which the subjects were most confident in recognizing. When correlations were examined just for the control and long-delay conditions (where the data points for the five ranks and features were based on responses significantly better than chance), it was found that they were higher for the ranked (by reaction time control) data than for the features results. Possibly, if the quality of salience is to embody quick, confident, and accurate processing, it is more aptly applied to feature ranks than to features.

The "difficult" features, defined by the reaction time control condition, tended to be more at risk when the task became harder. For example, the "easy" features in the short-exposure condition had fewer errors than chance but performance fell to guessing rate for the fourth and fifth ranks. The latencies for these "difficult" features were fast, indicating a speed-error tradeoff-a case where the results for the different measures do not positively covary. The data for the short-exposure condition suggests that the feature rank reflects the order of feature acquisition. The error data for the long-delay condition indicates that the higher ranked features were the first parts of the face to be lost. The absence of any interactions between the rank order, delay, or exposure duration, together with the significant positive correlations between error rates for the delay and short-exposure conditions, suggests that those features that are processed earliest are the best remembered. Consequently, it would appear that there is a single salience dimension which is both perceptual and mnemonic.

\section{Conclusion}

The above evidence suggests that in a specific case of picture recognition where global recognition cues are inadequate, Gestalt processing does not take place. A fixed-order, self-terminating feature-comparison strategy appears to be used, at least when making negative responses during the successive face recognition taskresults which corroborate Bradshaw and Wallace's (1971) findings for simultaneous face comparisons. Thus, subjects may sometimes make their decisions based on partial recognition information. There were some overall trends in the difficulty of specific feature processing. And the eyes and mouth benefited from immediate testing, possibly due to their role in conveying expression.

In a natural setting, we rarely see faces that are as similar as the faces used in this experiment, unless we meet monozygotic twins, so we may seldom need to make detailed feature comparisons when recognizing faces. However, this experiment shows that people are able to cope with picture recognition tasks that are too complex for a single diagnostic cue to suffice.

\section{REFERENCES}

ARgYle, M. Bodily communication. London: Methuen, 1975.

Bradshaw, J. L., \& Wallace, G. Models for the processing and identification of faces. Perception \& Psychophysics, 1971, 9. $443-448$.

Davies, G., Ellis, H., \& Shepherd, J. Cue saliency in faces as assessed by the 'Photofit' technique. Perception, 1977, 6, 263-269.

Ellis, H. D. Recognizing faces. British Journal of Psychology, 1975, 66, 409.426 .

Goldstein, A. G., \& Chance, J. E. Visual recognition memory for complex configurations. Perception \& Psychophysics, 1971, 9. 237-241.

Goldstein, A. G., \& Chance, J. Some factors in picture recognition memory. Journal of General Psychology, 1974, 90. 69-85.

Goldstein, A. G., \& Mackenberg, E. G. Recognition of human faces from isolated facial features: A developmental study. Psychonomic Science, 1966, 6, 149-150.

KIRK, R. E. Experimental design: Procedures for the behavioral sciences. Belmont, Calif: Brooks/Cole, 1968.

laughery, K. R., Fessler, P. K., Lenorovitz, D. R., \& Yoblick, D. A. Time delay and similarity effects in facial recognition. Journal of Applied Psychology, 1974, 59, 490-496.

Nickerson, R. S. Short-term memory for complex, meaningful, visual configurations: A demonstration of capacity. Canadian Journal of Psychology, 1965, 19, 155-160.

Phillips, W. A., \& Baddeley, A. D. Reaction time and shortterm visual memory. Psychonomic Science, 1971, 22, 73-74.

SCAPINEllo, K. F.. \& Y ARMEY, A. D. The role of familiarity and orientation in immediate and delayed recognition of pictorial stimuli. Psychonomic Science, 1970, 21, 329-331.

SHEPARD, R. N. Recognition memory for words, sentences, and pictures. Journal of Verbal Learning and Verbal Behavior, $1967,6,156-163$.

Smith. E. E., \& Nielsen. G. D. Representations and retrieval processes in short-term memory: Recognition and recall of faces. Journal of Experimental Psychology, 1970, 85, 397-405.

Standing, L., Conezio, J., \& Haber, R. N. Perception and memory for pictures: Single trial learning of 2500 visual stimuli. Psychonomic Science, 1970, 19, 73-74.

STERnBerg, S. Two operations in character recognition: Some evidence from reaction-time measurements. Perception \& Psychophysics, 1967, 2. 45-53.

STERnBerG, $S$. The discovery of processing stages: Extension of Donders' method. In W. G. Koster (Ed.), Attention and per- 
formance II. Amsterdam: North-Holland, 1969. (Reprinted from Acta Psychologica, 1969, 30.)

Townsend, J. T. A note of the identifiability of parallel and serial processes. Perception \& Psychophysics, 1971, 10, 161-163.

Walker-Smith, G. J., Gale, A. G., \& Findlay, J. M. Eye movement strategies involved in face perception. Perception, $1977,6,313-326$.

YARMEY, A. D. Recognition memory for familiar "public" faces: Effects of orientation and delay. Psychonomic Science, 1971, 24. $286-288$

\section{NOTE}

1. The Photo-fit Kit-Jacques Penry's Facial Identification Technique-is marketed by John Waddington of Kirkstall Ltd., Leeds, England.

(Received for publication November 1, 1977; revision accepted April 24, 1978.) 